\title{
Glucocorticoid receptor-mediated apoptosis in small-cell lung cancer requires interaction with BCL2
}

\author{
G Schlossmacher ${ }^{1}$, E Platt ${ }^{1}$, A Davies ${ }^{1}$, S Meredith ${ }^{1}$ and A White ${ }^{1,2}$ \\ ${ }^{1}$ Faculty of Life Sciences, Centre for Endocrinology and Diabetes ${ }^{2}$ Faculty of Medical and Human Sciences, \\ Manchester Academic Health Sciences Centre, University of Manchester, 3.016 AV Hill Building, \\ Manchester M13 9PT, UK
}

Correspondence should be addressed to A White

Email

anne.white@manchester.ac.uk

\begin{abstract}
Small-cell lung cancer (SCLC) tumours are highly aggressive. At the time of diagnosis,

patients have often developed metastases, and overall prognosis is particularly poor, making effective treatment difficult. Novel mechanisms need to be identified as treatment targets. We have previously found low levels of the glucocorticoid receptor (GR) in SCLC cell lines and demonstrated that over-expression of GR increases tumour cell death both in vitro and in vivo. We hypothesise that low levels of GR impair its inhibitory effect on BCL2 and thus provide a survival advantage to SCLC cell lines. The mechanism behind GR-induced apoptosis is currently unknown; therefore, pro- and anti-apoptotic genes were investigated for their role in GR-mediated apoptosis signalling. We found that over-expression of wtGR via retroviral transduction causes the DMS 79 SCLC cell line to undergo caspase-mediated apoptosis within $72 \mathrm{~h}$. Neither BAD nor BCL2L11 (BIM) mRNA and protein levels were affected by GR restoration implying that GR does not trigger apoptosis in the SCLC cell lines by up-regulating these pro-apoptotic genes. The anti-apoptotic $B C L 2$ gene was significantly overexpressed in six SCLC cell lines and the BCL2 inhibitor ABT-737 increased apoptosis in all three cell lines tested. GR interacted with BCL2 in DMS 153, DMS 79 and COR-L42 cell lines, suggesting that a protein interaction between GR and BCL2 could play a role in GR-induced apoptosis. A deeper understanding of the molecular mechanism for increasing GR expression in SCLC could provide novel treatment strategies in the future.
\end{abstract}

Key Words

\section{Introduction}

Lung cancer is the leading cause of cancer-related deaths in the Western world (Ihde 1995, Jemal et al. 2009). It is the most prevalent cancer in males in Britain and is second only to breast cancer in women. Small-cell lung cancer (SCLC) accounts for $10-15 \%$ of all lung cancers and tobacco smoking greatly increases the risk of developing SCLC (Riaz et al. 2012). It is the most aggressive form of lung cancer and has generally already metastasised by the time of diagnosis. The tumour becomes metastatic early in tumorigenesis with secondary cancers forming in the brain, liver and bone. The presence of these metastases means that surgery is often dismissed in favour of chemotherapy and/or radiotherapy. SCLC is initially highly sensitive to chemotherapy (Blackhall \& Shepherd 2007) but eventually relapses with a chemoresistant disease phenotype resulting in very poor prognosis (Jackman \& Johnson 2005). Even though considerable advances have been made in the diagnosis and treatment 
of SCLC in the past decade, long-term survival rate is still very low and therefore novel treatment approaches are required.

SCLC is a carcinoma of neuroendocrine origin and thus some tumours are able to secrete a variety of ectopic neuropeptides including the adrenocorticotropic hormone (ACTH) precursor, pro-opiomelanocortin (POMC; Stovold et al. 2011). In certain situations, this can give rise to the ectopic ACTH syndrome (Stewart et al. 1994). However, evidence suggests that SCLC tumours without clinical features of ectopic ACTH syndrome may secrete ACTH-related peptides (Stewart et al. 1994, Oliver et al. 2003, Stovold et al. 2013). The ectopic secretion of POMC by SCLC is resistant to normal negative feedback inhibition by glucocorticoids (Gcs), and this is the basis of the high-dose dexamethasone (Dex) suppression test in the diagnosis of Cushing's syndrome (Newell-Price et al. 2006).

The inability of Gcs to inhibit POMC expression in SCLC led to the investigation of the glucocorticoid receptor (GR) expression in SCLC cell lines. A multitude of factors can affect Gc sensitivity and we have previously shown that some human SCLC cell lines are resistant to Gcs and that this resistance is due to impaired GR expression (Farrell et al. 1993, Ray et al. 1994, 1996). More recently, we have found that a panel of SCLC cells have negligible amounts of functional GR due to GR promoter methylation and we hypothesised that this is an important factor contributing to Gc resistance (Kay et al. 2011). Restoration of GR expression in SCLC cells by transfection of exogenous GR is sufficient to restore Gc sensitivity (Ray et al. 1994). We have also shown that restoration of GR expression is sufficient to induce cell death by apoptosis in SCLC cells both in vitro (Sommer et al. 2007) and in vivo in a xenograft model (Sommer et al. 2010).

In lymphoid cells, Gcs can induce marked apoptosis; hence, they are used for the treatment of haematological malignancies. However, a canonical pathway for GR-induced apoptosis has not been identified and the mechanisms vary depending on cell origin (Herr et al. 2007).

Our previous work on GR-mediated apoptosis (Sommer et al. 2007, 2010) highlighted the effects of GR on members of the BCL2 family. The process of apoptosis is tightly regulated by both pro- and anti-apoptotic BCL2 family proteins. Regulation of apoptosis is achieved by BH3-only proteins (BAD, BIM, tBID, NOXA and PUMA), which will transmit death signals by interacting with anti-apoptotic family members (BCL2, BCL-xL and MCL-1), thereby inhibiting their cytoprotective function.
Abolishing anti-apoptotic signals allows BAX and BAK to be activated, where they play a part in forming pore complexes on the mitochondrial membrane resulting in the release of cytochrome $c$, which commits the cell to apoptosis (Adams \& Cory 2007, Sommer et al. 2010). Both sides of the BCL2 family balance activation and suppression of apoptosis initiation and this balance has been termed the 'BCL2 rheostat' (Ploner et al. 2008). The interaction between members of the BCL2 family is crucial to maintaining this balance and any effects leading to the up- or down-regulation of one of the pro- or anti-apoptotic members respectively can 'tip the balance' from a living cell to a cell undergoing apoptosis.

Our previous studies which showed that GR overexpression kills human SCLC cells in vitro and in vivo are extended here in order to elucidate the pathway of GR-induced apoptosis in SCLC cell lines. We show that anti-apoptotic BCL2 is overexpressed in our SCLC cell lines and that GR-mediated apoptosis does not signal via the pro-apoptotic genes BIM and BAD. Co-immunoprecipitation studies have identified an interaction between BCL2 and GR, suggesting that apoptosis signalling in response to GR over-expression is achieved by specific GR/BCL2 protein interactions and not through apoptotic gene regulation by GR.

\section{Subjects and methods}

\section{Cell culture and maintenance}

A549 human lung epithelial carcinoma cells, HEK293 human embryonic kidney cells and HeLa human cervical carcinoma cells (European Collection of Cell Cultures, Wiltshire, UK) were cultured in DMEM (Invitrogen) supplemented with 10\% FCS (Invitrogen).

Non-SCLC lines NCI-H358 and NCI-H727 (European Collection of Cell Cultures) and NCI-H23, NCI-H441 and NCI-H1299 (American Type Culture Collection, Manassas, VA, USA) were grown in RPMI-1640 medium (Invitrogen) supplemented with $10 \%$ FCS and $10 \mathrm{mM}$ HEPES as recommended by supplier.

SCLC cell lines used in this study were DMS 79, DMS 53 and DMS 153 and the 'COR' cell lines COR-L24, COR-L42, COR-L47, COR-L51 and COR-L103. All these cell lines were derived from patients with pathologically confirmed SCLC (Pettengill et al. 1980, Baillie-Johnson et al. 1985). All SCLC cell lines were cultured in RPMI-1640 medium (Invitrogen) supplemented with 10\% FCS and $10 \mathrm{mM}$ HEPES, as described previously (Ray et al. 1994).

Published by Bioscientifica Ltd. 
For treatment with the BCL2 inhibitor, cells were incubated for $48 \mathrm{~h}$ with $10 \mu \mathrm{M}$ ABT-737 made up in DMSO (kind gift from Abbott Laboratories) or DMSO alone as a vehicle control.

\section{Survival assay}

Survival rate of cell lines after treatment with ABT-737 was determined using the CellTiter-Glo Luminescent Cell Viability Assay (Promega) following the manufacturer's instructions. Luminescence was measured using a microplate luminometer.

\section{Immunoblot analysis}

Cells were lysed using $1 \times$ Chaps cell extract buffer (Cell Signalling Technology, Hitchin, UK) containing Complete protease inhibitor cocktail (Roche). Following three freeze/ thaw cycles, cell debris was pelleted by centrifugation and supernatants harvested. Protein concentration was measured using a NanoDrop. Samples were diluted in loading buffer (0.125 M Tris-Cl (pH 6.8), 0.1\% SDS, 20\% glycerol, $0.2 \%$ $\beta$-mercaptoethanol and $0.001 \%$ bromophenolblue) and analysed by SDS-PAGE on $4-12 \%$ Bis-Tris Novex gels (Invitrogen) and subsequent western blotting. The primary antibodies used were the mouse monoclonal anti-hGR (clone 41, 1:2500), which binds in the N-terminal region of GR (BD Biosciences, Oxford, UK), rabbit monoclonal anti-BAD (1:1000; Abcam, Cambridge, UK), rabbit polyclonal anti-BIM (1:2000; Merck Biosciences, Nottingham, UK), mouse monoclonal anti-BCL2 (1:2500; Abcam) and the mouse monoclonal $\alpha$-tubulin (1:5000; Sigma-Aldrich). Secondary antibodies were the HRP-conjugated anti-mouse IgG (1:5000) and anti-rabbit IgG (1:5000) (Invitrogen). To quantify protein levels, densitometric analysis was carried out using ImageJ Software (NIH, Bethesda, MD, USA) and normalised to the loading control ( $\alpha$-tubulin). This analysis was carried out on three separate blots for each experiment.

\section{RT-PCR}

RT-PCR was carried out using Omniscript RT-PCR Kit (Qiagen). RNA concentration of $2 \mu \mathrm{g} / 20 \mu \mathrm{l}$ of the reaction mixture was maintained for all the reactions. Unspecific anchored oligo d(T)20 primer (Invitrogen) was used for RT-PCR. Primer sequences for specific cDNA amplification were as follows: BIM (F, 5'-AGGTAGACAATTGCAGCCTGC-3'; R, 5'-CAATGCATTCTCCACACCAGG-3'), GR (F, 5'-GAGACGAATGAGAGTCCTTGG-3'; R, 5'-TGGAGGAGAGCTTACATCTGG-3') and GAPDH
(F, 5'-GCTTCGCTCTCTGCTC-3'; R, 5'-TCATACTTCTCATGGTTCACAC-3'). All primers were obtained from Eurofins MWG (London, UK).

\section{Retroviral infections}

Transduction of retroviral particles into SCLC cell lines was performed as detailed in Sommer et al. (2007). Briefly, HEK293 cells were transfected with plasmids for retroviral production, pFUNC1-eYFP/-GR-eYFP, Gag-Pol and VSV-G, using FuGene HD (Roche) at a 3:2 reagent:DNA ratio. Retrovirus production was stimulated by adding $10 \mathrm{mM}$ sodium butyrate $\geq 21 \mathrm{~h}$ after transfection. Target cells were mixed with virus-containing supernatant and centrifuged at $1000 \boldsymbol{g}$ for $2 \mathrm{~h}$ in the presence of $8 \mu \mathrm{g} / \mathrm{ml}$ Polybrene (Millipore, Watford, UK) on two consecutive days. Posttransduction, cells were cultured in normal growth media containing $10 \%$ serum for 24,48 or $72 \mathrm{~h}$.

\section{Immunocytochemistry}

Analysis of GR Cells were pelleted, fixed for $24 \mathrm{~h}$ in $10 \%$ formalin and embedded in paraffin wax. Sections were cut at $5 \mu \mathrm{m}$ and mounted on polysine-coated slides. Antigen retrieval was performed on all slides in pH6 citrate buffer at $95^{\circ} \mathrm{C}$ for $30 \mathrm{~min}$. The slides were blocked and then stained with GR-specific antibody (BD Biosciences) at $5 \mu \mathrm{g} / \mathrm{ml}$ and visualised by DAB staining protocol (Chromagen Envision System, Dako, Cambridge, UK). Cell nuclei were counterstained with haematoxylin. Negative controls used an IgG1 non-specific primary antibody also at $5 \mu \mathrm{g} / \mathrm{ml}$. Imaging was conducted using an Axioskop brightfield microscope and Axiovision Software (Zeiss Microscopy \& Imaging, Cambridge, UK).

For PARP p85 staining DMS 79 cells expressing GR-eYFP or eYFP were applied to poly-L-lysine-coated coverslips and fixed with $4 \%$ formaldehyde. Cells were permeabilised with PBS/0.2\% Triton X-100. After washing, cells were blocked in PBS/0.1\% Tween 20+5\% donkey serum. Anti-PARP p85 Fragment pAb (Promega) diluted 1:250 in blocking buffer was added to cells and incubated overnight. Secondary antibody incubation was performed in the dark using Alexa Fluor 546 donkey anti-rabbit IgG (Invitrogen) diluted 1:500 in PBS. Coverslips were mounted using ProLong Gold with DAPI (Invitrogen).

Images were collected on an Olympus BX51 upright microscope using a $60 \times / 1.40$ UPlanApo objective and captured using a Coolsnap ES camera (Photometrics, Tucson, AZ, USA) through MetaVue Software (Molecular Devices, Sunnyvale, CA, USA). Specific band pass filter sets for DAPI,

Published by Bioscientifica Ltd 
FITC and Texas red were used to prevent bleed through from one channel to the next. Images were processed and analysed using ImageJ (http://rsb.info.nih.gov/ij).

\section{Co-immunoprecipitation studies}

Dynabeads Protein G (25 $\mu$; Invitrogen) were incubated with $5 \mu$ goat polyclonal anti-Bcl-2 antibody (Santa Cruz) in $250 \mu \mathrm{l}$ PBS-T (0.1\% Tween 20). The antibody was cross linked to the beads using bis(sulfosuccinimidyl)suberate $\left(\mathrm{BS}^{3}\right.$; Thermo Fisher Scientific, Loughborough, UK) according to the manufacturer's instructions. The following day, the beads were pelleted and washed with PBS-T. Ten micrograms of total protein extract was incubated with the antibody-bound beads for $2 \mathrm{~h}$ on an orbital shaker at room temperature. Upon removal of supernatant, the beads were washed three times with $300 \mu$ ice-cold PBS-T. After the final wash, the beads were re-suspended in $100 \mu \mathrm{l}$ PBS-T and transferred to a fresh tube. The protein complexes of interest were eluted by addition of $60 \mu \mathrm{l}$ $2 \times$ Laemmli sample buffer followed by heating at $95^{\circ} \mathrm{C}$ for $10 \mathrm{~min}$. Eluted sample protein was analysed by SDS-PAGE followed by western blot for GR protein.

\section{Statistical analysis}

Statistical analysis was carried out using GraphPad Prism 6 (Graphpad, La Jolla, CA, USA). Independent Student's $t$-test was performed to determine significance.

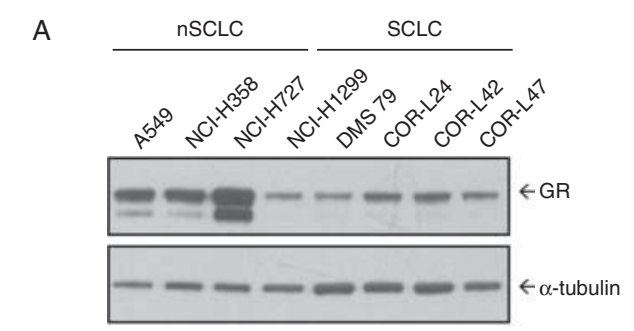

C
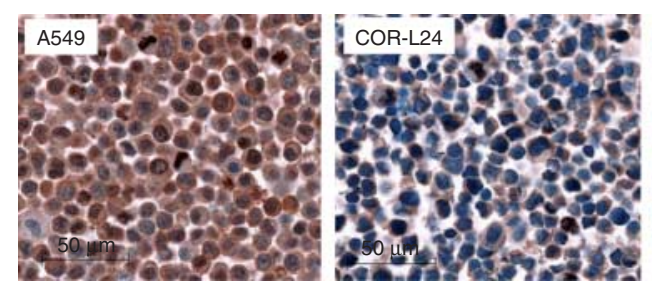

\section{Results}

\section{GR expression is reduced in human SCLC cell lines}

GR protein was investigated by western blot analysis in the SCLC cell lines, DMS 79, COR-L24, COR-L42 and COR-L47, and compared with the Gc-sensitive cell line, A549, and three additional non-SCLC (nSCLC) cell lines, NCI-H358, NCI-H727 and NCI-H1299. Overall, the SCLC cell lines have reduced levels of GR protein compared with the A549 cells and two of the other nSCLC cells (Fig. 1A and B). Immunocytochemical analysis of GR emphasises the differences in the relative amounts of GR between the SCLC cell lines (Fig. 1C). In addition, COR L24 cells have compound heterozygous mutations in the GR, and one allele has a mutation in the DNA binding domain resulting in a $50 \%$ reduction in activity of these receptors (Ray et al. 1996).

\section{Restoration of GR expression induces PARP cleavage}

Expression of a GR-eYFP transgene introduced into DMS 79 cells by retroviral infection resulted in apoptosis as evidenced by increased levels of the cleaved PARP fragment p85 in the SCLC cells (Fig. 2A). As PARP is a direct substrate of caspase 3 , increased levels of cleaved PARP are a reflection of caspase 3 activity within these cells. Apoptosis was observed at 48 and $72 \mathrm{~h}$ post-infection with the maximum occurring at $72 \mathrm{~h}$ (Fig. 2B).
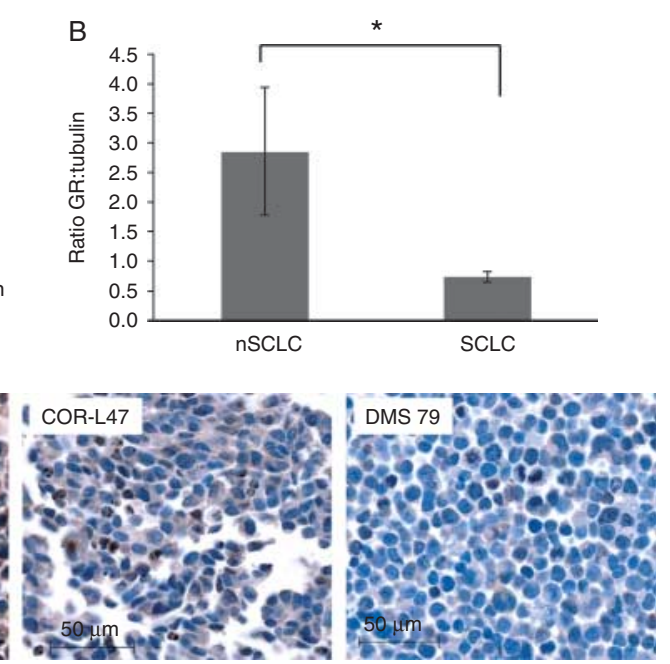

Figure 1

Endogenous GR levels in SCLC cells are low compared with nSCLC. (A and B) Representative western blot for glucocorticoid receptor (GR) and $\alpha$-tubulin in a panel of SCLC and non-SCLC cell lines. (B) Averaged protein levels for SCLC and non-SCLC cell lines as calculated from (A). Western blots are representative of three independent experiments; ${ }^{*} P<0.05(n=3)$. (C) Immunostaining of GR (brown) in the SCLC cell lines COR-L24, COR-L47, DMS 79 and the non-SCLC control cell line A549. Nuclear staining in blue.

Published by Bioscientifica Ltd. 

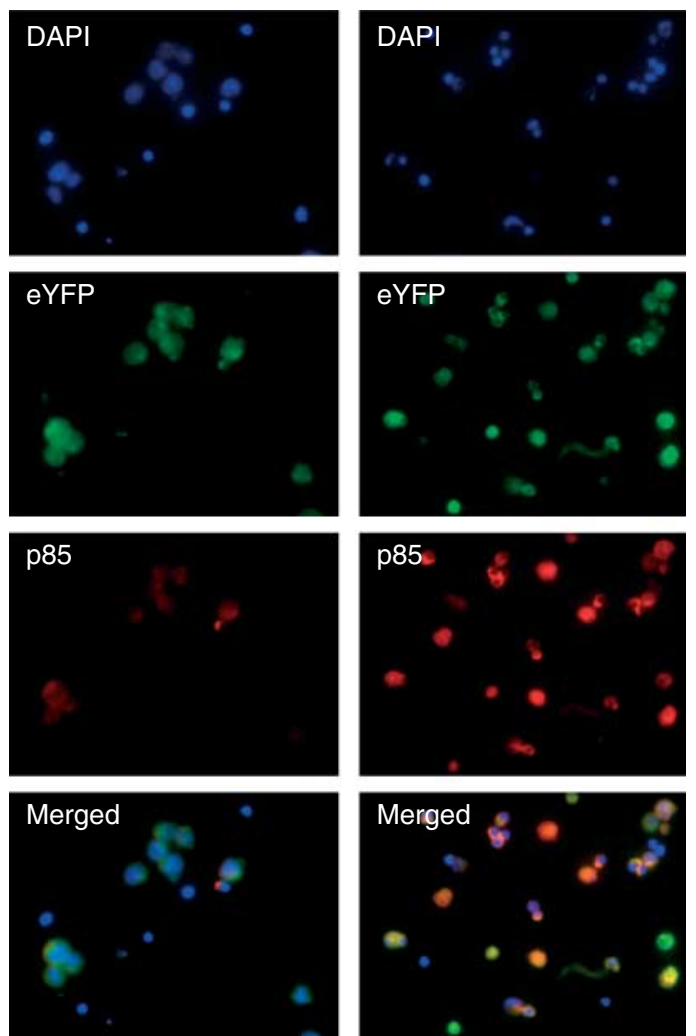

B

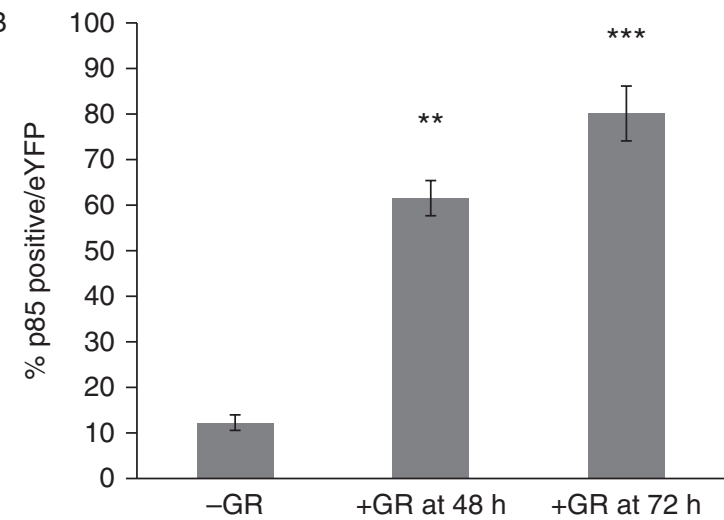

Figure 2

Restoring GR in SCLC cells causes apoptosis. (A) Cells transduced with GR-eYFP or eYFP were stained for the $\mathrm{p} 85$ fragment, which results from PARP cleavage (red) and then analysed for co-localisation with eYFP (green). (B) Percentage of cells positive for p85 and eYFP. Cell counts derived from three independent infections and represented as percentage of p85 positive to eYFP positive; $* * P<0.01$ and $* * * P<0.001(n=3)$.

\section{BIM is not a target of GR signalling in SCLC cells}

One of the most well-known activators of the caspase cascade is the pro-apoptotic 'Bcl2-like protein 11' $\mathrm{BIM}$ (O'Connor et al. 1998). Transcription of the BIM gene is increased during Dex-induced apoptosis in acute lymphoblastic leukaemia (ALL) cells, lymphoma cells and mouse thymocytes (Wang et al. 2003, Abrams et al. 2004, Zhao et al. 2007). However, the exact mechanism is largely unknown.

Over-expression of GR-eYFP by retroviral transduction into DMS 79 cells (Fig. 3) caused BIM transcript levels to decrease after $72 \mathrm{~h}$. Interestingly, Dex treatment has no effect on any of the BIM transcription levels (Fig. 3A). GR-eYFP in the presence or absence of Dex had no impact on BIM protein levels at any of the time points (Fig. 3B) even though the presence of GR-eYFP was confirmed by western blots, indicating that retroviral transduction was successful.

\section{Endogenous GR is up-regulated in response to exogenous GR expression}

The western blot for GR in DMS 79 cells transduced with GR-eYFP or eYFP indicated an increase in endogenous GR in the GR-eYFP-infected cells (Fig. 3B and C) such that GR-eYFP protein was present at $\sim 60 \%$ of the endogenous GR level. In addition, RT-PCR for GR transcripts also showed an increase in GR expression at the mRNA level (Fig. 3D).

\section{Pro-apoptotic BAD is not a mediator of GR-induced apoptosis in SCLC cells}

Given the role of BAD as another important pro-apoptotic protein, we wanted to determine whether it had a role in GR-mediated apoptosis. We determined expression levels of the pro-apoptotic protein BAD in seven SCLC cell lines. BAD protein levels were significantly higher in the SCLC cell lines DMS 153 and COR-L42 but were unchanged in any of the other SCLC cell lines under basal conditions (Fig. 4A and B) where we have previously shown low GR expression.

To ascertain whether restoring wtGR functionality increased transcriptional activity of the $B A D$ gene leading to a higher abundance of BAD protein in the cells, DMS 79 cells were retrovirally transduced with either the pFUNC1GR-eYFP or eYFP constructs.

No differences were observed in BAD protein levels after 48 or $72 \mathrm{~h}$ in transduced cells with restored GR vs control-transduced cells. Transduction itself was successful as evidenced by the increase in GR levels compared with the vector control, but BAD protein levels did not differ between sample groups (Fig. 4C and D). 
A $24 \mathrm{~h}$ $48 \mathrm{~h}$ $72 \mathrm{~h}$

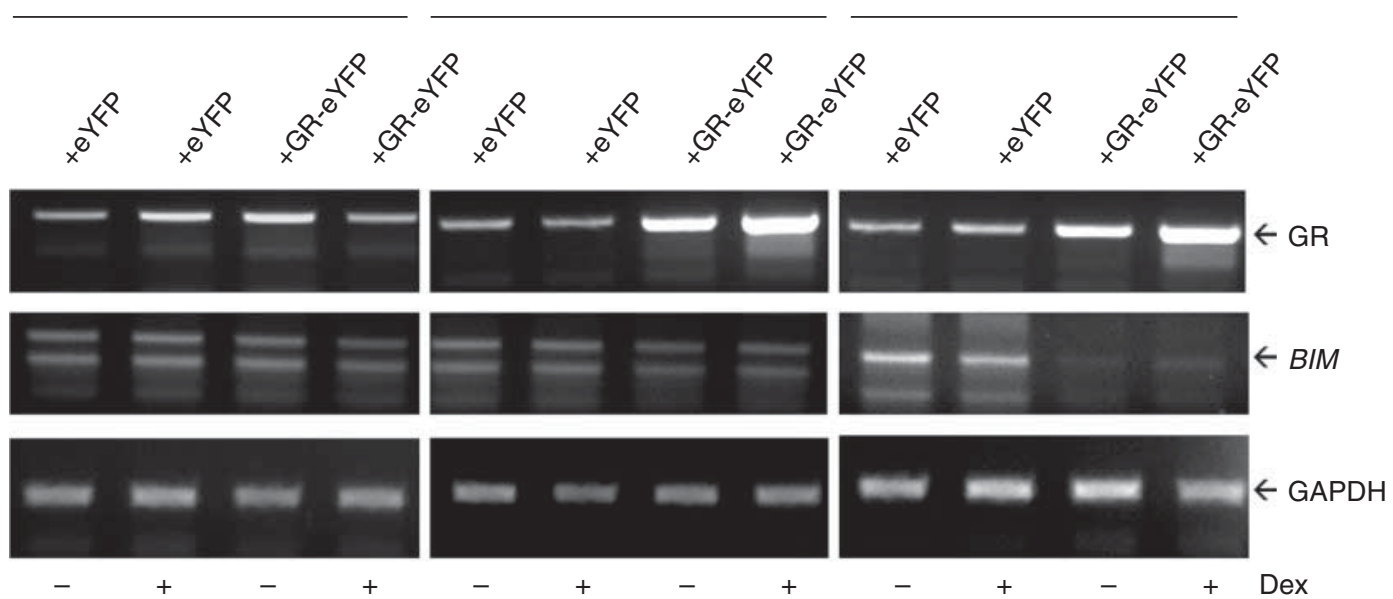

B

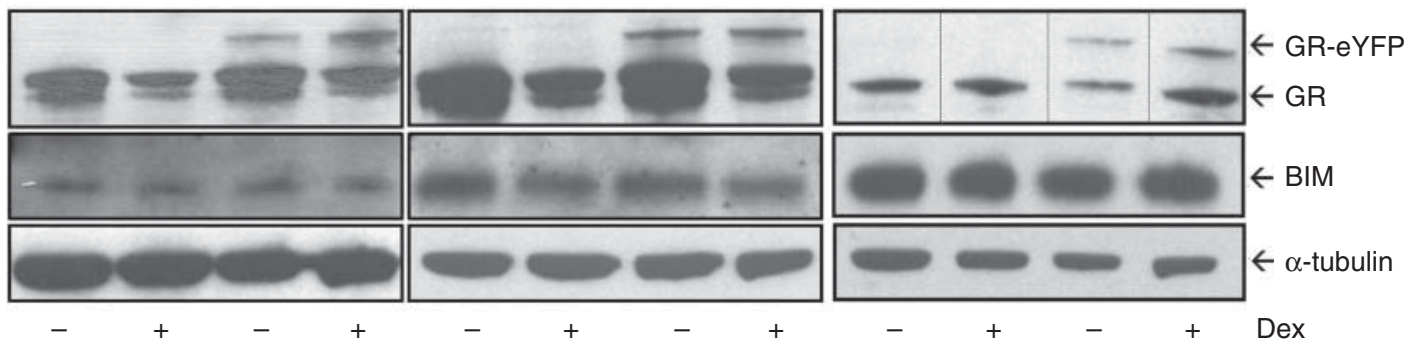

C

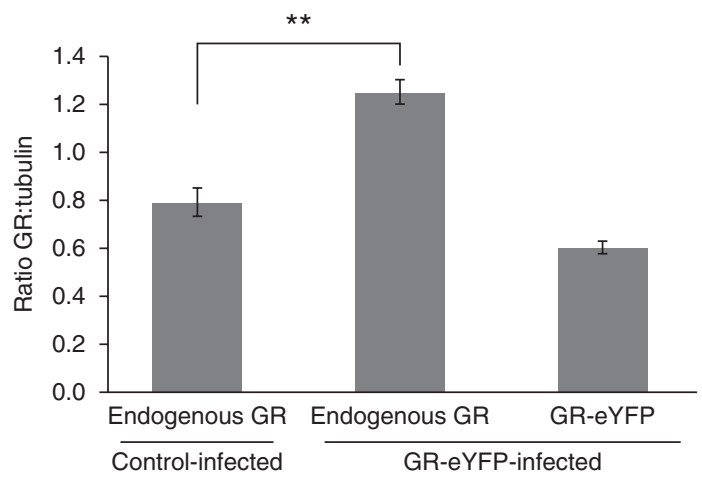

D

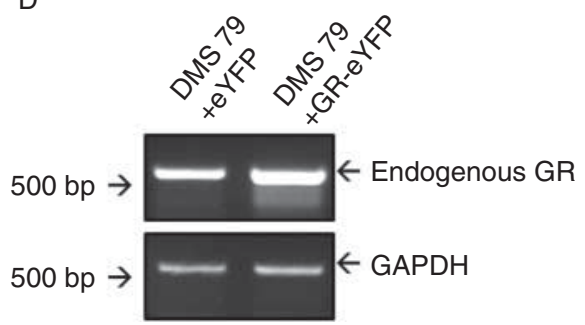

\section{Figure 3}

GR does not alter expression of BIM and endogenous GR levels increase in response to exogenous GR over-expression. DMS 79 cells were retrovirally infected with eYFP or GR-eYFP and treated with DMSO or $100 \mathrm{nM}$ Dex for up to $72 \mathrm{~h}$. (A) mRNA levels were determined via RT-PCR using gene-specific primers for BIM. (B) Protein sizes: GR (94 kDa), GR-eYFP (110 kDa), BIM

\section{SCLC cell lines have increased levels of BCL2}

In six of the seven SCLC cell lines tested, BCL2 is significantly overexpressed when compared with HEK293 and A549 cells (Fig. 5A and B). DMS 79 cells retrovirally infected with GR-eYFP or eYFP were also analysed to determine the effect of GR restoration on BCL2 up to $72 \mathrm{~h}$ after treatment (Fig. 5C). Increasing the
(23 kDa) and $\alpha$-tubulin (50 kDa). (C) GR-eYFP or eYFP analysed $24 \mathrm{~h}$ post-infection by densitometry. (D) RT-PCR for GR mRNA transcripts. Images are representative of three independent over-expression experiments; ${ }^{*} P<0.01(n=3)$.

level of GR by retroviral infection had no effect on BCL2 expression and Dex treatment of the GR-eYFP cells did not elicit a response.

\section{SCLC cell lines require BCL2 to suppress apoptosis}

Inhibition of BCL2 with the BH3-mimetic inhibitor ABT-737 decreased cell survival significantly in all the

Published by Bioscientifica Ltd. 
A
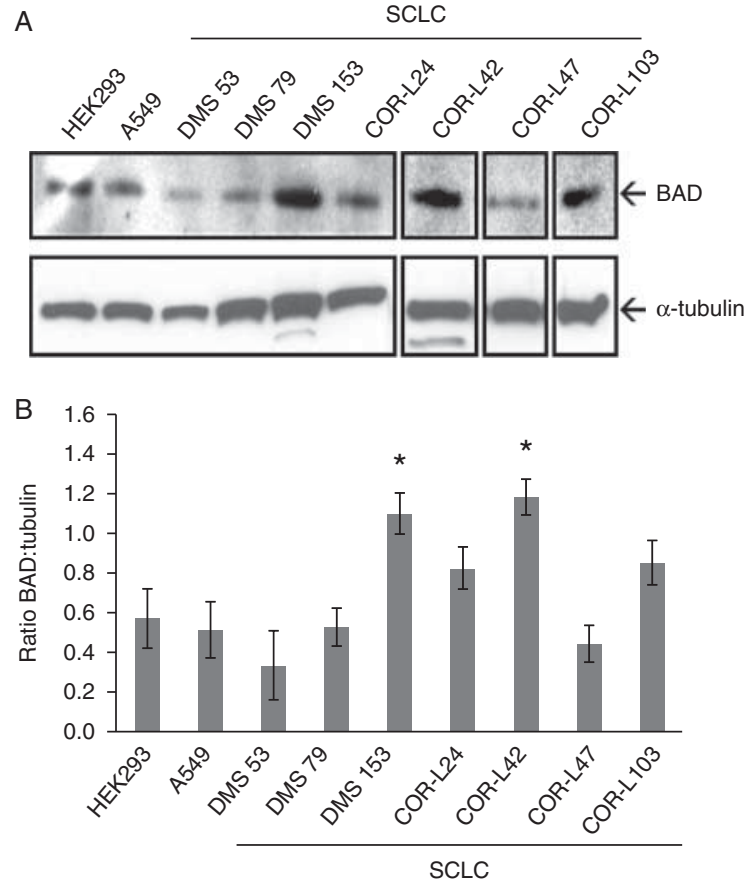

C
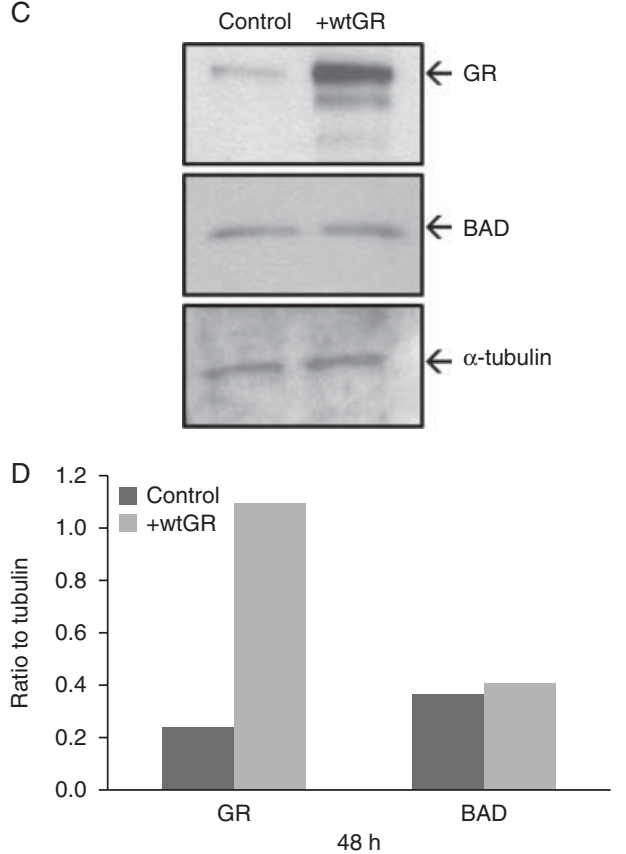

Figure 4

GR restoration does not affect BAD expression. Protein levels of $B A D$ in SCLC cell lines were measured by western blot (A) and quantified by densitometry. (B) DMS 79 cells transfected with wtGR or control vector were analysed for BAD and GR protein levels (C) and were quantified by densitometry. (D) Total cell protein was analysed $48 \mathrm{~h}$ post-transfection. Protein sizes: BAD (23 kDa), GR $\alpha$ (94 kDa) and $\alpha$-tubulin (50 kDa). Western blot images are representative of three independent experiments. Results are presented relative to HEK293 and A549 cells as controls; $* P<0.05(n=3)$. three cell lines after 48-h treatment (Fig. 6A). Cleaved caspase 3 was also increased in all the three cell lines after ABT-737 treatment (Fig. 6B). Given the clear evidence that BCL2 inhibition drives all the SCLC cell lines into apoptosis, it would appear that the SCLC cell lines are dependent on BCL2 over-expression to suppress apoptosis.

\section{BCL2 interacts with GR in three SCLC cell lines}

We hypothesised that GR could interact with BCL2, thereby impairing its anti-apoptotic properties and eliciting an increased apoptotic response. To test this, we undertook co-immunoprecipitation studies on DMS 153, DMS 79 and COR-L42 cell protein extracts. Using magnetic beads with cross linked antibody to precipitate BCL2, we demonstrated that endogenous GR was indeed able to form a complex with BCL2 in all the three cell lines (Fig. 7). Interestingly, no interaction was detected with GR-eYFP in the cell lines.

\section{Discussion}

We have previously shown low endogenous GR expression in a panel of SCLC cell lines, which we attributed to methylation of the GR promoter. Over-expression of GR in these SCLC cell lines leads to apoptotic cell death (Kay et al. 2011). The current study used the retroviral delivery approach to rescue low GR expression levels in DMS 79 cells in order to investigate the mechanism underlying the GR-induced apoptosis in SCLC cell lines. Although the increase in GR did not affect the pro-apoptotic genes BIM and $B A D$ or the anti-apoptotic BCL2 gene expression, an interaction between GR and BCL2 proteins was observed.

Previous studies carried out by our group both in vitro (Sommer et al. 2007) and in a mouse xenograft model in vivo (Sommer et al. 2010) have demonstrated that overexpression of GR by retroviral transduction increases apoptosis. The mechanism by which GR can initiate apoptosis has only been studied in cell lines derived from lymphoid malignancies (Herr et al. 2003, Leis et al. 2004, Zhu et al. 2004) and there are still cell-type-specific differences (Reichardt et al. 2000, Schmidt et al. 2004). Therefore, we wanted to investigate which downstream pathway is involved in triggering GR-mediated apoptosis in SCLC cell lines.

It is difficult to envisage how to specifically up-regulate GR in SCLC cells as a therapeutic approach, but it may be possible to identify a possible therapeutic target in the apoptotic pathway downstream of GR. Therefore, it is important to identify the specific apoptotic pathway

Published by Bioscientifica Ltd 
A

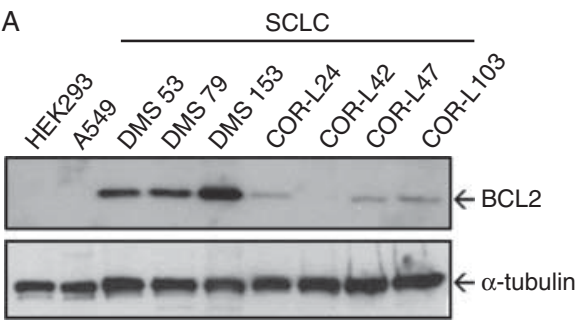

C
B

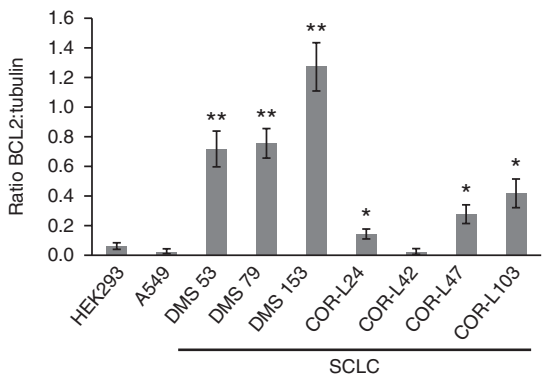

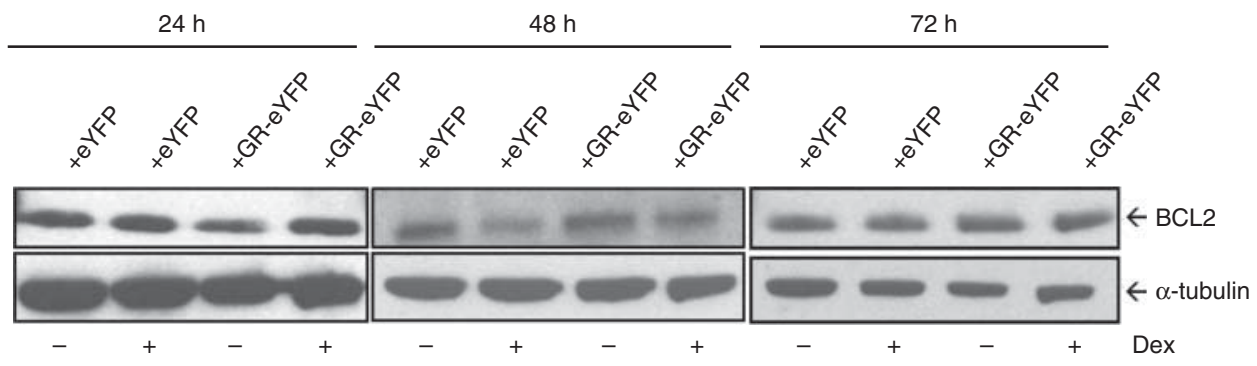

Figure 5

$B C L 2$ is overexpressed in SCLC but is unaffected by GR. Levels of BCL2 in SCLC cell lines as measured by (A) western blot and (B) densitometry. HEK293 and A549 cell lines act as non-SCLC controls. (C) DMS 79 cells were retrovirally infected with eYFP or GR-eYFP and treated with DMSO or

activated by GR treatment. We chose the BIM gene as a target for apoptotic signalling as it has been shown that its up-regulation plays an important role in Dex-induced apoptosis of ALL cells, lymphoma cells and mouse thymocytes (Wang et al. 2003, Abrams et al. 2004, Zhao et al. 2007). However, in our study, no changes in $B I M$ were seen in cells transduced with GR-eYFP and treated with Dex until the 72 -h time point when GR-eYFP-containing cells unexpectedly showed decreased amounts of BIM mRNA.

We also observed that introduction of exogenous wtGR caused endogenous GR to increase at both the mRNA and protein level. To date, the mechanism for this phenomenon is unknown, but studies have identified glucocorticoid response units (GRUs) within the GR gene, suggesting that GR can auto-up-regulate its expression (Geng et al. 2008). Currently, it is believed that binding of GR and c-MYB to GRUs found in GR exon 1 promoter regions $1 \mathrm{~A}, 1 \mathrm{C}$ and $1 \mathrm{D}$ up-regulates GR expression, while binding of GR and ETS down-regulates GR expression (Geng et al. 2008, Geng \& Vedeckis 2011). However, these studies are limited to ALL cells and may not be relevant in SCLC cells. Further investigations into the mechanisms underlying this form of GR auto-regulation are required, as up-regulation of GR expression may be able to restore Gc
100 nM Dex for up to $72 \mathrm{~h}$. Protein sizes: BCL2 (25 kDa), $\alpha$-tubulin (50 kDa). Western blot images are representative of three independent experiments; $* * P<0.01$ and $* P<0.05(n=3)$.

sensitivity to SCLC cells, thereby increasing their susceptibility to pro-apoptotic signalling.

An alternative target for GR in apoptotic signalling could be the $B A D$ gene. In a panel of seven SCLC cell lines analysed for BAD protein expression, none of them had lower expression than the non-SCLC cell lines, suggesting that BAD may not be affected by the low GR levels in these cell lines. Furthermore, while overexpressing wtGR increased GR protein levels, BAD levels remained unchanged between control and wtGR-transfected samples. Increased transcript levels do not always correlate with increased protein product, so we cannot rule out a post-transcriptional mechanism blocking the translation of $B A D$ mRNA.

BCL2 was a likely candidate as a GR target, as it is often overexpressed in cancers (Reed 1995, Kroemer 1997, Yip \& Reed 2008). In the majority of the SCLC cell lines analysed, BCL2 protein was significantly overexpressed compared with control cell lines. Increased expression of BCL2 would provide SCLC cells with a survival advantage as BCL2 is able to block the pro-apoptotic proteins from triggering cytochrome c release from the mitochondria. This suggestion was supported by the fact that inhibiting BCL2 with ABT-737 decreases cell number and increases apoptosis, indicating that increased levels of BCL2 may

Published by Bioscientifica Ltd 
A
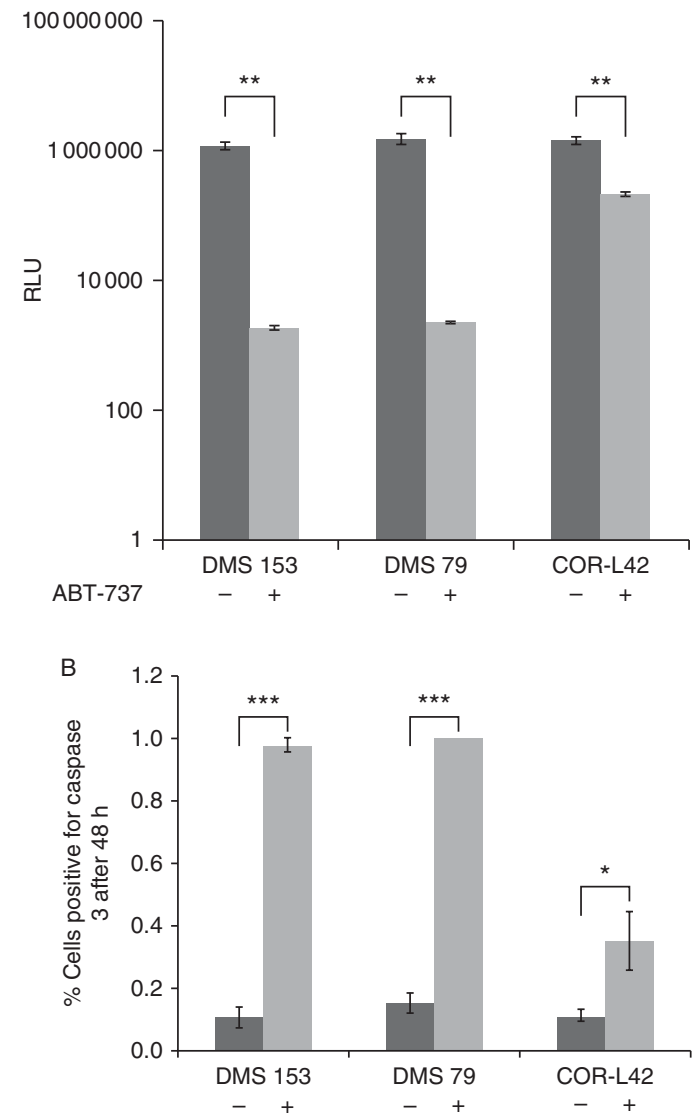

Figure 6

Inhibition of BCL2 in SCLC cells drives apoptosis. Apoptosis in three SCLC cell lines as measured by (A) ATP viability assay and (B) caspase 3 activation. Cell lines were chosen based on their BCL2 expression levels (DMS 153, high; DMS 79, intermediate and COR-L42, low). Cells were treated with DMSO or $10 \mu \mathrm{M}$ ABT-737 for $48 \mathrm{~h}$. For (B), 100 cells per slide were counted and represented as percentage of caspase 3-positive to total cells; $* * * P<0.001, * * P<0.01$ and $* P<0.05(n=3)$. promote survival of the cancer cells. Herr et al. (2003) showed that in lymphocytes, Gc treatment decreases BCL2 gene expression. However, in our study, increasing GR expression did not decrease BCL2 protein levels and therefore does not explain the mechanism whereby GR is increasing cell death in the SCLC cell lines.

As none of the potential genomic apoptosis targets selected for this study responded to the over-expression of wtGR within the SCLC cell lines, we hypothesised that GR may exert its effect in a non-genomic manner. There is an interaction between GR and BCL2 in neurons whereby GR sequesters BCL2 and this is predicted to prevent BCL2 from blocking pro-apoptotic factors (Du et al. 2009). Based on our findings that BCL2 is overexpressed in SCLC, we hypothesised that an interaction between GR and BCL2 in the SCLC cell lines may have a similar effect and increase apoptosis. This was supported by our finding that an interaction between BCL2 and GR occurred in all three SCLC cell lines tested (DMS 153, DMS 79 and COR-L42) after retroviral transduction with GR-eYFP. Interestingly, the BCL2 interaction was only observed with the endogenous GR protein and not with the GR-eYFP fusion protein. It may be that the eYFP tag is partially blocking the binding of the GR antibody and therefore it is not possible to detect the interaction with BCL2. This would also explain why GR-eYFP levels appear to be low when compared with endogenous GR. It is also important to note that the expression profiles for exogenous GR-eYFP in our experiments can only provide a snapshot of GR-eYFP levels prior to cell death. The SCLC cell lines grow as compact floating aggregates and require two cycles of infection of GR-eYFP, so expression will be spread over $72 \mathrm{~h}$, as will the time of cell death. However, it is also possible that the eYFP tag is altering the affinity of GR binding to BCL2, thus preventing the formation of a

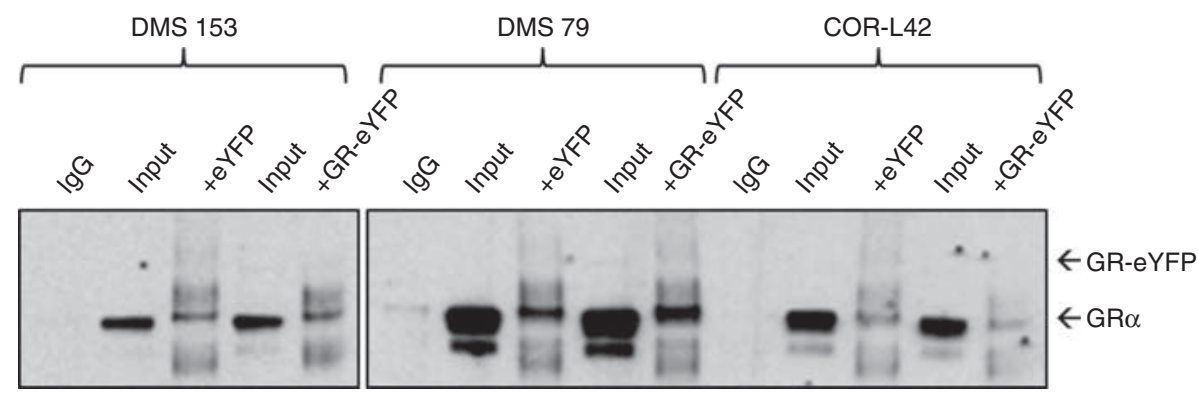

Figure 7

BCL2 can interact with endogenous GR but not GR-eYFP. DMS 79, DMS 153 and COR-L42 cells were retrovirally infected with eYFP or GR-eYFP and harvested after $48 \mathrm{~h}$. Total protein was extracted and used for Co-IP experiments using BCL2 for IP and the resulting pulldown pool blotted for GR. Arrows indicate GR (94 kDa) or GR-eYFP (110 kDa) protein bands. http://erc.endocrinology-journals.org DOI: 10.1530/ERC-13-0402
(C) 2013 Society for Endocrinology Printed in Great Britain
Published by Bioscientifica Ltd. 
stronger interaction between GR and BCL2. Nevertheless, this process is resulting in significant apoptosis, mediated by the over-expression of GR-eYFP.

In summary, restoring GR expression in the DMS 79 SCLC cell line elicits apoptosis not by up-regulating proapoptotic or down-regulating anti-apoptotic signals but by interacting with BCL2 either directly or as part of a larger complex. As BCL2 is overexpressed in SCLC cell lines, we believe that the interaction with GR blocks its cytoprotective effects, thereby facilitating cytochrome c release and subsequent activation of the caspase cascade. Further investigation into mechanisms up-regulating endogenous GR levels may provide a viable treatment for SCLC in the future.

\section{Declaration of interest}

The authors declare that there is no conflict of interest that could be perceived as prejudicing the impartiality of the research reported.

\section{Funding}

Both G Schlossmacher and E Platt are funded by a BBSRC studentship award and a Barbara Mawer Endowment studentship. This work was supported by the Manchester Biomedical Research Centre. Abbott Laboratories kindly provided ABT-737.

\section{Acknowledgements}

The authors are grateful to Dr A Stevens for helpful discussions.

\section{References}

Abrams MT, Robertson NM, Yoon K \& Wickstrom E 2004 Inhibition of glucocorticoid-induced apoptosis by targeting the major splice variants of BIM mRNA with small interfering RNA and short hairpin RNA. Journal of Biological Chemistry 279 55809-55817. (doi:10.1074/jbc. M411767200)

Adams JM \& Cory S 2007 The Bcl-2 apoptotic switch in cancer development and therapy. Oncogene 26 1324-1337. (doi:10.1038/sj.onc.1210220)

Baillie-Johnson H, Twentyman PR, Fox NE, Walls GA, Workman P, Watson JV, Johnson N, Reeve JG \& Bleehen NM 1985 Establishment and characterisation of cell lines from patients with lung cancer (predominantly small cell carcinoma). British Journal of Cancer 52 495-504. (doi:10.1038/bjc.1985.220)

Blackhall FH \& Shepherd FA 2007 Small cell lung cancer and targeted therapies. Current Opinion in Oncology 19 103-108. (doi:10.1097/CCO. Ob013e328011bec3)

Du J, Wang Y, Hunter R, Wei Y, Blumenthal R, Falke C, Khairova R, Zhou R, Yuan P, Machado-Vieira R et al. 2009 Dynamic regulation of mitochondrial function by glucocorticoids. PNAS 106 3543-3548. (doi:10.1073/pnas.0812671106)

Farrell WE, Stewart MF, Clark AJ, Crosby SR, Davis JR \& White A 1993 Glucocorticoid inhibition of ACTH peptides: small cell lung cancer cell lines are more resistant than pituitary corticotroph adenoma cells. Journal of Molecular Endocrinology 10 25-32. (doi:10.1677/jme.0. 0100025)
Geng CD \& Vedeckis WV 2011 A new, lineage specific, autoup-regulation mechanism for human glucocorticoid receptor gene expression in 697 pre-B-acute lymphoblastic leukemia cells. Molecular Endocrinology 25 44-57. (doi:10.1210/me.2010-0249)

Geng CD, Schwartz JR \& Vedeckis WV 2008 A conserved molecular mechanism is responsible for the auto-up-regulation of glucocorticoid receptor gene promoters. Molecular Endocrinology 22 2624-2642. (doi:10.1210/me.2008-0157)

Herr I, Ucur E, Herzer K, Okouoyo S, Ridder R, Krammer PH, von Knebel Doeberitz M \& Debatin KM 2003 Glucocorticoid cotreatment induces apoptosis resistance toward cancer therapy in carcinomas. Cancer Research 63 3112-3120.

Herr I, Gassler N, Friess H \& Büchler MW 2007 Regulation of differentia pro- and anti-apoptotic signaling by glucocorticoids. Apoptosis 12 271-291. (doi:10.1007/s10495-006-0624-5)

Ihde DC 1995 Small cell lung cancer. State of the art therapy 1994. Chest 107 243S-248S. (doi:10.1378/chest.107.6_Supplement.243S)

Jackman DM \& Johnson BE 2005 Small-cell lung cancer. Lancet 366 1385-1396. (doi:10.1016/S0140-6736(05)67569-1)

Jemal A, Siegel R, Ward E, Hao Y, Xu J \& Thun MJ 2009 Cancer statistics. CA: A Cancer Journal for Clinicians 59 225-249. (doi:10.3322/caac.20006)

Kay P, Schlossmacher G, Matthews L, Sommer P, Singh D, White A \& Ray D 2011 Loss of glucocorticoid receptor expression by DNA methylation prevents glucocorticoid induced apoptosis in human small cell lung cancer cells. PLOS ONE 6 e24839. (doi:10.1371/journal.pone.0024839)

Kroemer G 1997 The proto-oncogene Bcl-2 and its role in regulating apoptosis. Nature Medicine 3 614-620. (doi:10.1038/nm0697-614)

Leis H, Page A, Ramírez A, Bravo A, Segrelles C, Paramio J, Barettino D, Jorcano JL \& Pérez P 2004 Glucocorticoid receptor counteracts tumorigenic activity of Akt in skin through interference with the phosphatidylinositol 3-kinase signaling pathway. Molecular Endocrinology 18 303-311. (doi:10.1210/me.2003-0350)

Newell-Price J, Bertagna X, Grossman AB \& Nieman LK 2006 Cushing's syndrome. Lancet 367 1605-1617. (doi:10.1016/S0140-6736(06) 68699-6)

O'Connor L, Strasser A, O'Reilly LA, Hausmann G, Adams JM, Cory S \& Huang DC 1998 Bim: a novel member of the Bcl-2 family that promotes apoptosis. EMBO Journal 17 384-395. (doi:10.1093/emboj/17.2.384)

Oliver RL, Davis JR \& White A 2003 Characterisation of ACTH related peptides in ectopic Cushing's syndrome. Pituitary 6 119-126. (doi:10.1023/B:PITU.0000011172.26649.df)

Pettengill OS, Sorenson GD, Wurster-Hill DH, Curphey TJ, Noll WW, Cate CC \& Maurer LH 1980 Isolation and growth characteristics of continuous cell lines from small-cell carcinoma of the lung. Cancer $\mathbf{4 5}$ 906-918. (doi:10.1002/1097-0142(19800301)45:5<906::AIDCNCR2820450513>3.0.CO;2-H)

Ploner C, Rainer J, Niederegger H, Eduardoff M, Villunger A, Geley S \& Kofler R 2008 The BCL2 rheostat in glucocorticoid-induced apoptosis of acute lymphoblastic leukemia. Leukemia 22 370-377. (doi:10.1038/ sj.leu.2405039)

Ray DW, Littlewood AC, Clark AJ, Davis JR \& White A 1994 Human small cell lung cancer cell lines expressing the POMC gene have aberrant glucocorticoid receptor function. Journal of Clinical Investigation 93 1625-1630. (doi:10.1172/JCI117143)

Ray DW, Davis JR, White A \& Clark AJ 1996 Glucocorticoid receptor structure and function in glucocorticoid-resistant small cell lung carcinoma cells. Cancer Research 56 3276-3280.

Reed JC 1995 Regulation of apoptosis by bcl-2 family proteins and its role in cancer and chemoresistance. Current Opinion in Oncology 7 541-546. (doi:10.1097/00001622-199511000-00012)

Reichardt HM, Umland T, Bauer A, Kretz O \& Schütz G 2000 Mice with an increased glucocorticoid receptor gene dosage show enhanced resistance to stress and endotoxic shock. Molecular and Cellular Biology 20 9009-9017. (doi:10.1128/MCB.20.23.9009-9017.2000) 
Riaz SP, Lüchtenborg M, Coupland VH, Spicer J, Peake MD \& Møller H 2012 Trends in incidence of small cell lung cancer and all lung cancer. Lung Cancer 75 280-284. (doi:10.1016/j.lungcan.2011.08.004)

Schmidt S, Rainer J, Ploner C, Presul E, Riml S \& Kofler R 2004 Glucocorticoid-induced apoptosis and glucocorticoid resistance: molecular mechanisms and clinical relevance. Cell Death and Differentiation 11 S45-S55. (doi:10.1038/sj.cdd.4401456)

Sommer P, Le Rouzic P, Gillingham H, Berry A, Kayahara M, Huynh T, White A \& Ray DW 2007 Glucocorticoid receptor expression exerts an anti-survival effect on human small cell lung cancer cells. Oncogene 26 1-11. (doi:10.1038/sj.onc.1210524)

Sommer P, Cowen RL, Berry A, Cookson A, Telfer BA, Williams KJ, Stratford IJ, Kay P, White A \& Ray DW 2010 Glucocorticoid receptor over-expression promotes human small cell lung cancer apoptosis in vivo and thereby slows tumor growth. Endocrine-Related Cancer 17 203-213. (doi:10.1677/ ERC-09-0241)

Stewart PM, Gibson S, Crosby SR, Penn R, Holder R, Ferry D, Thatcher N, Phillips P, London DR \& White A 1994 ACTH precursors characterize the ectopic ACTH syndrome. Clinical Endocrinology 40 199-204. (doi:10.1111/j.1365-2265.1994.tb02468.x)

Stovold R, Blackhall F, Meredith S, Hou J, Dive C \& White A 2011 Biomarkers for small cell lung cancer: neuroendocrine, epithelial and circulating tumour cells. Lung Cancer 76 263-268. (doi:10.1016/j. lungcan.2011.11.015)

Stovold R, Meredith SL, Bryant JL, Babur M, Williams KJ, Dean EJ, Dive C, Blackhall FH \& White A 2013 Neuroendocrine and epithelial phenotypes in small-cell lung cancer: implications for metastasis and survival in patients. British Journal of Cancer 108 1704-1711. (doi:10.1038/bjc.2013.112)

Wang Z, Malone MH, He H, McColl KS \& Distelhorst CW 2003 Microarray analysis uncovers the induction of the proapoptotic BH3-only protein BIM in multiple models of glucocorticoid-induced apoptosis. Journal of Biological Chemistry 278 23861-23867. (doi:10.1074/jbc. M301843200)

Yip KW \& Reed JC 2008 Bcl-2 family proteins and cancer. Oncogene $\mathbf{2 7}$ 6398-6406. (doi:10.1038/onc.2008.307)

Zhao YN, Li Q \& Yang K 2007 Role of pro-apoptotic protein bim in mouse thymocytes apoptosis induced by dexamethasone. Sichuan Da Xue Xue Bao. Yi Xue Ban 38 851-854.

Zhu XY, Liu YJ, Lu J \& Xu RB 2004 Knockdown of glucocorticoid receptor expression by RNA interference promotes cell proliferation in murine macrophage RAW264.7 cells. Journal of Steroid Biochemistry and Molecular Biology 92 375-382. (doi:10.1016/j.jsbmb. 2004.07.010)

Received in final form 9 September 2013

Accepted 11 September 2013

Made available online as an Accepted Preprint

13 September 2013
(C) 2013 Society for Endocrinology Printed in Great Britain
Published by Bioscientifica Ltd. 